\title{
Male Partner's Involvement in HIV Counselling and Testing and Associated Factors among Partners of Pregnant Women in Gondar Town, Northwest Ethiopia
}

\author{
Alemu Zenebe, ${ }^{1}$ Abebaw Gebeyehu, ${ }^{2}$ Lemma Derseh, ${ }^{3}$ and Kedir Y. Ahmed ${ }^{4}$ \\ ${ }^{1}$ Department of Pediatric Operation Theatre, Black Lion teaching Hospital, Addis Ababa, Ethiopia \\ ${ }^{2}$ Department of Reproductive Health, Institute of Public Health, College of Medicine and Health Science, \\ University of Gondar, P.O. Box 196, Gondar, Ethiopia \\ ${ }^{3}$ Department of Epidemiology and Biostatistics, Institute of Public Health, College of Medicine and Health Science, \\ University of Gondar, P.O. Box 196, Gondar, Ethiopia \\ ${ }^{4}$ Department of Public Health, College of Medicine and Health Science, Debre Markos University, P.O. Box 269, Debre Markos, Ethiopia
}

Correspondence should be addressed to Kedir Y. Ahmed; kedirymam331@gmail.com

Received 19 November 2015; Accepted 10 July 2016

Academic Editor: Fabio Facchinetti

Copyright @ 2016 Alemu Zenebe et al. This is an open access article distributed under the Creative Commons Attribution License, which permits unrestricted use, distribution, and reproduction in any medium, provided the original work is properly cited.

Background. Despite the existence of several programmes promoting male involvement in HIV counselling and testing during their wife's pregnancy as a part of PMTCT, few men have heeded the call. The aim of this study was to assess male partner's involvement in HCT and its associated factors. Methods. This study was based on institution based cross-sectional study design that used systematic random sampling technique. A total of 416 partners were interviewed in the data collection. Multivariable logistic regression model was fitted to identify the independent predictors. Result. In this study, the prevalence of male involvement in HCT was found to be $40.1 \%$ (95\% CI: 35.3\%-44.7\%). The independent predictors of male involvement were partners who were younger, were cohabitant, were with multigravida wives, were knowledgeable on route of mother-to-child transmission, and discussed HCT. Conclusion. The prevalence of male involvement in HCT was found to be suboptimal compared to similar studies in Ethiopia. There is a need of interventions on partners who are older, separated, and with lower gravidity wife. Awareness creation campaign should also be created on the route of mother-to-child transmission of HIV and on the importance of discussion with wife.

\section{Introduction}

HIV pandemic created an enormous challenge to the survival of mankind worldwide [1]. Worldwide there are an estimated 33.3 million people infected with HIV; Sub-Saharan Africa bears the greater burden with an estimated 22.5 million people infected with HIV [2]. According to UNAIDS, women represent $52 \%$ of those infected with HIV worldwide and in Sub-Saharan Africa $60 \%$ of those infected with HIV are women [2]. With a national adult HIV prevalence of $1.5 \%$ (1.9\% in women and $1.0 \%$ in men), Ethiopia is one of the country's most severely hit by the epidemic [3].

Mother-to-child transmission (MTCT) is an important source of HIV infection among Ethiopian children, which accounts for more than $90 \%$ of pediatric AIDS [1, 4].
Prevention of Mother-to-Child Transmission (PMTCT) programmes have been proven to be effective in reducing the risk of HIV transmission from infected mothers to their children [5]. Without intervention, the risk of MTCT of HIV ranges from $20 \%$ to $45 \%$. With specific interventions in nonbreastfeeding populations, the risk of MTCT can be reduced to less than $2 \%$ and to $5 \%$ or less in breastfeeding populations [6]. Antenatal care (ANC) is a major entry point for PMTCT programmes especially in countries with a high prevalence of HIV. It creates an opportunity to capture pregnant mothers and their male partners to reverse the transmission of HIV during pregnancy, labour, and breastfeeding [7]. Male involvement is necessary for improving women's uptake of core PMTCT services; it is a key contributor to community acceptance and support of PMTCT [8]. 
However, actual involvement of male partners in PMTCT programmes in several counties of Sub-Saharan Africa is low and programmes report difficulties in attracting the involvement of male partners. Studies conducted in African countries showed that male involvement in PMTCT during pregnancy ranges from $11 \%$ to $58.3 \%$ [5, 9-11]. In Ethiopia, studies conducted in Arba Minch and Debre Markos town reported that $53.6 \%$ and $55.4 \%$ of partners accompanied their wives for HIV testing and counselling $[12,13]$.

Despite the existence of several programmes promoting male involvement in HIV counselling and testing during their wives' pregnancy as a part of PMTCT, few men have heeded the call. Numerous reasons for nonparticipation of male partners in HIV counselling and testing as PMTCT programmes have been gained from studies that primarily focused on women as respondents. This study primarily focused on men to gain understanding of factors that influence their involvement in these programmes. Thus, this study was aimed at assessing male involvement and associated factors in HCT among partners of pregnant women in Gondar town, Northwest Ethiopia.

\section{Methods}

2.1. Study Design and Study Setting. Institution based crosssectional study was used to assess the prevalence and associated factors of male involvement in HCT among partners of pregnant women in Gondar administrative city from July to November 2014. Gondar is an ancient town located $727 \mathrm{Km}$ North of Addis Ababa with an estimated population of 207,044 of which $50.8 \%$ were females and the rest are males. There are eight health centers that provide ANC service with HCT of both pregnant women and their husbands.

2.2. Source and Study Population. Partners of all pregnant women who did voluntary HCT in the current pregnancy were the study population. Those partners selected by systematic random sampling technique were included in this study. Partners who are unable to communicate for different reasons and those who are not living together were excluded from the study.

2.3. Sample Size Determination and Sampling Procedure. By using single population proportion formula, as there is no information about it, about $50 \%$ prevalence of male involvement was used. Considering 5\% margin of error and $95 \%$ confidence interval with $10 \%$ nonresponse rate, the final minimum sample size was found to be 422 . Based on the number of mothers who were on ANC follow-up, the samples were distributed to all eight health centers proportionate to their number. After the first participant was selected using lottery method, systematic random sampling technique was used to select eligible participants.

2.4. Data Collection. Data was collected by using pretested structured questionnaire. The tool was developed in English and translated to Amharic and then back to English to check for its consistency. Ten data collectors and three supervisors who can speak Amharic language were recruited. Partners involved in HCT were interviewed at the facility level and those who did not involve in HCT were at the household level. To assure the quality of the data, three-day training was given to data collectors and supervisors, and on each data collection day some percent of the collected data were reviewed by principal investigator; any problems faced in the time of data collection were discussed and immediate solution was made.

2.5. Operational Definitions. Operational definitions are as follows:

(i) Male involvement: when husbands or partners of pregnant women attended both HIV counselling and testing (HCT) during ANC visit for the purpose of PMTCT service.

(ii) Knowledge about route of MTCT: when respondents know at least one route of transmission of MTCT of HIV from three questions.

(iii) Knowledge about PMTCT: when respondents know at least one way of PMTCT from three questions.

2.6. Data Processing and Analysis. The data were checked, coded, and entered to Epi-info software version 3.5.1 and cleaning was performed by using SPSS 20. Frequencies, proportions, and measures of central tendency and variation were used to describe the study participant. Binary logistic regression was used to examine association between dependent and each independent variable. All variables with $p<0.2$ in bivariate analysis were entered into multiple logistic regression model to identify factors independently associated with male involvement in HCT during pregnancy. Backward stepwise likelihood ratio was used to select the final independent predictors. The significance of Odds Ratios (OR) was determined with $95 \%$ CI and $p<0.05$.

2.7. Ethical Consideration. Ethical clearance was obtained from University of Gondar, and letter of permission was obtained from the respected health institutions. Informed consent from each study participant was being obtained after explaining the purpose of the study. Confidentiality of the information was assured by omitting names from the questionnaire and maximum effort was made to maintain privacy of the respondents during the interview. No question was asked about their serostatus. No incentives were provided to the respondents as a way of motivating them to participate in the study. All the identified potential participants agreed to participate in the study.

\section{Result}

3.1. Sociodemographic Characteristics of Partners. The nonresponse rate of this study was found to be $6(1.42 \%)$. The mean $( \pm \mathrm{SD})$ age of partners who participated in this study was 34.38 years $( \pm 6.0)$. The majority, $210(50.5 \%)$ of them, reported the current pregnancy as wife's first pregnancy (Table 1).

Among respondents, 206 (49.5\%) reported that the average distance of the living area from health institution used to perform HCT was less than five Km. Regarding the amount 
TABLE 1: A sociodemographic characteristic of partners of pregnant women in Gondar town, Northwest Ethiopia, 2013.

\begin{tabular}{|c|c|c|}
\hline Variables & Frequency & Percentage (\%) \\
\hline \multicolumn{3}{|l|}{ Age } \\
\hline $20-29$ & 79 & 19 \\
\hline $30-39$ & 237 & 57 \\
\hline $40+$ & 100 & 24 \\
\hline \multicolumn{3}{|l|}{ Marital statues } \\
\hline Married & 403 & $96.9 \%$ \\
\hline Unmarried & 13 & $3.1 \%$ \\
\hline \multicolumn{3}{|l|}{ Religion } \\
\hline Orthodox christian & 287 & 69 \\
\hline Muslim & 97 & 23.3 \\
\hline Others (protestant or catholic) & 32 & 7.7 \\
\hline \multicolumn{3}{|l|}{ Educational status } \\
\hline No formal education & 66 & 15.9 \\
\hline Primary education & 97 & 23.3 \\
\hline Secondary and above & 253 & 60.8 \\
\hline \multicolumn{3}{|l|}{ Occupation } \\
\hline Private business & 143 & 34.4 \\
\hline Government employee & 88 & 21.2 \\
\hline NGO & 74 & 17.8 \\
\hline Daily labor & 62 & 14.9 \\
\hline Farmer and others & 49 & 11.8 \\
\hline \multicolumn{3}{|l|}{ Wife's number of pregnancies } \\
\hline 1 & 166 & 39.9 \\
\hline $2-3$ & 210 & 50.5 \\
\hline $4-5$ & 34 & 8.2 \\
\hline $6+$ & 6 & 1.4 \\
\hline \multicolumn{3}{|l|}{ Time of living together } \\
\hline Not living together & 11 & 2.6 \\
\hline $1-5$ years & 254 & 61.1 \\
\hline $6-10$ years & 108 & 26 \\
\hline $11-15$ years & 33 & 7.9 \\
\hline $16+$ years & 10 & 2.4 \\
\hline \multicolumn{3}{|l|}{ Distance to nearby health facility } \\
\hline Less than $5 \mathrm{~km}$ & 206 & 49.5 \\
\hline $5-10 \mathrm{~km}$ & 128 & 30.8 \\
\hline More than $10 \mathrm{~km}$ & 82 & 19.7 \\
\hline \multicolumn{3}{|l|}{ Money paid for transportation } \\
\hline Did not pay & 34 & 8.2 \\
\hline$<5$ Ethiopian birr & 182 & 43.8 \\
\hline 5-10 Ethiopian birr & 165 & 39.6 \\
\hline$>10$ Ethiopian birr & 35 & 8.4 \\
\hline
\end{tabular}

of money they paid for transports, 182 (43.8\%) of the them paid less than five Ethiopian birr for the trip (Table 1).

3.2. Knowledge and Attitude towards PMTCT. About 386 $(92.8 \%)$ of them knew at least one route of MTCT and 301 (78\%), 298 (77.2\%), and 199 (51.6\%) of them reported MTCT of HIV during pregnancy, breastfeeding, and childbirth, respectively. The majority, 384 (92.3\%) of respondents, knew
TABLE 2: Knowledge and attitude towards PMTCT and risk perception of male partners of pregnant women in Gondar town, Northwest Ethiopia, 2013.

\begin{tabular}{|c|c|c|}
\hline Variables & Frequency & $\begin{array}{c}\text { Percentage } \\
(\%)\end{array}$ \\
\hline \multicolumn{3}{|l|}{ Occurrence of MTCT $(n=386)$} \\
\hline During pregnancy & 301 & 78 \\
\hline During childbirth & 199 & 51.6 \\
\hline During breastfeeding & 298 & 77.7 \\
\hline \multicolumn{3}{|l|}{ PMTCT methods $(n=384)$} \\
\hline ART & 324 & 84.4 \\
\hline Caesarean section & 13 & 3.4 \\
\hline Avoiding of breastfeeding & 221 & 57.6 \\
\hline \multicolumn{3}{|l|}{ Presence of HCT during ANC visit } \\
\hline Yes & 375 & 90.1 \\
\hline No & 16 & 3.9 \\
\hline I do not know & 25 & 6 \\
\hline \multicolumn{3}{|l|}{ Necessity of partner testing } \\
\hline Yes & 396 & 95.2 \\
\hline No & 20 & 4.8 \\
\hline \multicolumn{3}{|l|}{ Discordant test result } \\
\hline Yes & 356 & 85.6 \\
\hline No & 60 & 14.4 \\
\hline \multicolumn{3}{|l|}{$\begin{array}{l}\text { Feeling when seen with pregnant women } \\
\text { in clinic }\end{array}$} \\
\hline Feel nothing & 214 & 51.4 \\
\hline Feel happiness & 197 & 47.4 \\
\hline Feel ashamed & 5 & 1.2 \\
\hline \multicolumn{3}{|l|}{ Risk perception } \\
\hline Yes & 98 & 23.6 \\
\hline No & 318 & 76.4 \\
\hline \multicolumn{3}{|l|}{ Reasons of risk perception $(n=98)$} \\
\hline Used unsterile sharp object & 57 & 58.2 \\
\hline Had multiple sexual partner & 44 & 44.9 \\
\hline Had sexual contact without condom & 39 & 39.8 \\
\hline Had sex with positive person & 10 & 10.2 \\
\hline
\end{tabular}

at least one method of PMTCT and 324 (84.4\%) of them knew that provision of ARTs for the mother could help to reduce MTCT of HIV. On the other hand, 221 (57.6\%) of them knew that avoiding breastfeeding is one of the alternatives for preventing HIV transmission from mother to child, but only 3.4\% [12] of respondents were aware that risk of MTCT of HIV could be reduced by caesarean section. Among respondents, 375 (90.1\%) of them knew the presence of HCT for pregnant women during their ANC visit. Three hundred ninety-six (95.2\%) of them agreed on the necessity of partner testing. About 356 (85.6\%) of partners knew that discordant result can be found among married partners (Table 2).

3.3. Perceived Risk to Acquire the Virus. Perceived risk of acquiring the virus was reported in about 98 (23.6\%) of male 
TABLE 3: Level of involvement of partners of pregnant women towards HCT in Gondar town, Northwest Ethiopia, 2013.

\begin{tabular}{|c|c|c|}
\hline Variables & Frequency & $\begin{array}{l}\text { Percentage } \\
(\%)\end{array}$ \\
\hline \multicolumn{3}{|c|}{ Discussed about HCT with their wife } \\
\hline Yes & 278 & 66.8 \\
\hline No & 138 & 33.2 \\
\hline \multicolumn{3}{|c|}{$\begin{array}{l}\text { Willingness to visit PMTCT clinic with } \\
\text { his wife }\end{array}$} \\
\hline Yes & 288 & 69.2 \\
\hline No & 128 & 30.8 \\
\hline \multicolumn{3}{|c|}{ Visited PMTCT clinic with his wife } \\
\hline Yes & 212 & 51 \\
\hline No & 204 & 49 \\
\hline \multicolumn{3}{|l|}{ Involved in counselling only } \\
\hline Yes & 174 & 41.8 \\
\hline No & 242 & 58.2 \\
\hline \multicolumn{3}{|l|}{$\begin{array}{l}\text { Involved for both counselling and } \\
\text { testing }\end{array}$} \\
\hline Yes & 167 & 40.1 \\
\hline No & 249 & 59.9 \\
\hline \multicolumn{3}{|l|}{$\begin{array}{l}\text { Reasons for partners involvement } \\
(n=167)\end{array}$} \\
\hline Feel responsibility & 100 & 59.9 \\
\hline Initiated by provider & 65 & 38.9 \\
\hline Initiated by wife & 44 & 26.3 \\
\hline \multicolumn{3}{|c|}{$\begin{array}{l}\text { Reasons for partners noninvolvement } \\
(n=249)\end{array}$} \\
\hline Work overload & 131 & 52.6 \\
\hline Fear of acquiring the virus & 116 & 46.6 \\
\hline Confidentiality issue & 47 & 18.9 \\
\hline Fear of inaccurate test result & 12 & 4.8 \\
\hline
\end{tabular}

partners. Of these, 57 (58.2\%) used unsterile sharp object and $44(44.9 \%)$ of them had multiple sexual partners (Table 2).

3.4. Level of Male Involvement in HCT. Among partners, 278 (66.8\%) discussed HCT with their pregnant wives. And more than two-thirds (69.2\%) of them had willingness to accompany PMTCT clinic with their pregnant wife together. Two hundred twelve (51\%) of them visited PMTCT clinic with their wife. About 174 (41.8\%) of respondents were involved in the counselling part only and $167(40.1 \%)$ of them with 95\% CI (35.2-44.2) participated in both counselling and testing, meaning the overall involvement in HCT (Table 3). Among those involved in HCT, 100 (59.9\%) were involved because they felt responsibility. Work overload which was mentioned by 131 (52.6\%) of them was the main reason for noninvolvement of partners (Table 3 ).

3.5. Factors Associated with Male Involvement in HCT. In multivariable analysis, being at younger age group, couples living together, wife's number of pregnancies, having knowledge on the timing of MTCT, and husbands discussing HCT with their wives were positively and significantly associated with male involvement in HCT. The odds of male involvement in HCT was higher in 20-29-year (AOR = 4.94 95\% CI: $1.97-12.39$ ) and $30-39$ years' (AOR = 2.82 95\% CI: $1.37-5.81$ ) age groups as compared to those who were 40 years of age and above.

Those partners who are living together with their wives were 5.5 (95\% CI: 1.97-15.39) times more likely to be involved in HCT. Wife's gravidity of 2-3 (AOR $=5.34$ 95\% CI: 1.38 20.69) and 4-5 (AOR $=8.10$ 95\% CI: $1.52-43.32)$ was significantly associated with male involvement in HCT compared to those who had only one pregnancy. The likelihood of male involvement in HCT was found to be higher $(\mathrm{AOR}=7.41$ 95\% CI: 1.80-30.45) among those who knew the timing of mother-to-child transmission. Those partners who reported discussion about HCT with their wives had increased (AOR $=8.60$ 95\% CI: 4.30-17.21) odds of involvement in HCT compared to their counterparts (Table 4).

\section{Discussion}

In this study, about $40.1 \%$ of the partners escorted their wives to ANC and received HIV counselling and testing together. This finding is relatively similar to study conducted in Addis Ababa and Tanzania community which was $44 \%$ and $46.3 \%$, respectively $[9,14]$. However, the finding is lower than that reported in Cameroon which was 58.3\% [5], while it was higher than pooled estimate of studies conducted in India, Cameroon, Georgia, and the Dominican Republic which was $36.1 \%$ and another study conducted in Cape Town, South Africa, which was $32 \%[11,15]$. Thus, the finding of this study implies that there is already an encouraging platform for male involvement in the study area, and this could serve as a springboard to achieve full scale male involvement in HCT in the city of Gondar and other similar urban areas.

This study demonstrated that the level of male involvement in HCT was found to be higher among younger male partners. This finding is consistent with reviewed literatures which found that males involved in HCT were younger than those who received HCT alone in the same clinic [16]. This might be due to increased communication between couples and level of knowledge expected to be reduced with age. This is supported by study conducted in South Africa, 23.5\% of individuals 50 years of age and above did not know the route of transmission of HIV from mother to child [17]. Moreover, an operational research conducted in Zimbabwe showed that as age increased majority of men fear going for HIV tests [18]. However, similar studies conducted in Cameroon and Western Uganda showed that the proportion of males accompanying their partner increased with age, for example, in rural western Uganda males older than 35 years were 2.89 times more likely to receive VCT than those of 35 years or younger $[5,19]$. The difference with these studies could be explained by the existence of social support and difference in health service utilization in the later studies.

This result showed that male partners of pregnant women who were living with their wives were significantly more likely to be tested than those partners of pregnant women living in separated place. Absence of the male partner for 
TABLE 4: Factors associated with male involvement in HCT in Gondar town, Northwest Ethiopia, 2013.

\begin{tabular}{|c|c|c|c|c|}
\hline \multirow{2}{*}{ Variables } & \multicolumn{2}{|c|}{ Male involvement } & \multirow{2}{*}{ COR with 95\% CI } & \multirow{2}{*}{ AOR with 95\% CI } \\
\hline & Yes & No & & \\
\hline \multicolumn{5}{|l|}{ Age } \\
\hline $20-29$ & 36 & 43 & $2.51(1.33,4.73)$ & $4.94(1.97,12.39)^{* *}$ \\
\hline $30-39$ & 106 & 131 & $2.43(1.44,4.08)$ & $2.82(1.37,5.81)^{*}$ \\
\hline $40+$ & 25 & 75 & 1 & 1 \\
\hline \multicolumn{5}{|l|}{ Living together } \\
\hline Yes & 161 & 199 & $6.74(2.82,16.12)$ & $5.50(1.97,15.39)^{* *}$ \\
\hline No & 6 & 50 & 1 & 1 \\
\hline \multicolumn{5}{|c|}{ Wife's total pregnancy } \\
\hline One & 66 & 100 & 1 & 1 \\
\hline Two-three & 87 & 123 & $1.07(0.71,1.62)$ & $5.34(1.38,20.69)^{*}$ \\
\hline Four-five & 13 & 21 & $0.94(0.44,2.0)$ & $8.10(1.52,43.32)^{*}$ \\
\hline Six and above & 1 & 5 & $0.30(0.04,2.65)$ & $1.35(0.09,20.23)$ \\
\hline \multicolumn{5}{|c|}{ Knew time of MTCT } \\
\hline Yes & 164 & 222 & $6.65(1.98,22.29)$ & $7.41(1.80,30.45)^{*}$ \\
\hline No & 3 & 27 & 1 & 1 \\
\hline \multicolumn{5}{|l|}{ Discussed HCT } \\
\hline Yes & 153 & 125 & $10.84(5.94,19.77)$ & $8.60(4.30,17.21)^{*}$ \\
\hline No & 14 & 124 & 1 & 1 \\
\hline
\end{tabular}

discussion at home and decreased likelihood of accompanying his pregnant wife during ANC follow-up could be the possible explanation for the above finding. In this study, male involvement in HCT was significantly associated with wife's number of pregnancies. This could be explained by the fact that for each additional pregnancy there is increased frequency of contact of mothers with health care workers which increases their awareness and their chance of discussion with their husbands. Furthermore, these findings contradict with studies conducted in India and Kenya and showed that males who had fewer children were more likely to assist their partner in pregnancy and childbirth than males who had large number of children [20, 21].

Those partners who know at least one mode of transmission of HIV from MTC were 7.4 times more involved in HCT compared to their counterparts. This finding is similar to the study conducted in Zambia which showed that knowledge and the total score on level of involvement were positively and significantly associated [22]. This might be due to increased level of knowledge and awareness about HCT expected to have a positive influence on men's involvement in HCT. Having history of discussion about HIV testing with pregnant wife remained significantly associated with male attendance at the antenatal clinic for HCT. This finding was similar to another study conducted in Zambia [22]. Having discussion with their wives might help them to get what they heard from the health care workers during their wives' ANC visit which could be the possible explanation for increased uptake of partners.

The possibility of social desirability bias due to sensitiveness of issues and cross-sectional nature of the study which fails to show causal relationship were among the limitations of this study. Study was conducted both in health institution and in community level to address both involvers and noninvolvers which is the strength of this study.

\section{Conclusion}

Despite the existence of several programmes promoting male involvement in HIV counselling and testing during their wives' pregnancy as a part of PMTCT, still lower proportion of them accompany their wives for HCT. The prevalence of male involvement was found to be significantly higher among partners who are younger, living with their wives, are living with multigravida wives, are knowledgeable about mode of mother-to-child transmission of HIV, and discussed HCT with their wives. Therefore, there is a need of an intervention in the independent predictors.

\section{Competing Interests}

The authors declared that they have no conflict of interests.

\section{Acknowledgments}

The authors would like to forward their thanks to all supervisors and data collectors for their willingness and timely submission of questionnaires. Their thanks also go to University of Gondar for their library service and academic support. In addition, they thank Gondar health office and all health institutions for facilitation of data collection. 


\section{References}

[1] Federal HIV/AIDS Prevention and Control Office, Federal Ministry of Health: Guidelines for Prevention of Mother-to-Child Transmission of HIV in Ethiopia, Federal HIV/AIDS Prevention and Control Office, Addis Ababa, Ethiopia, July 2007.

[2] WHO, Global Report on the Global AIDS Epidemic. Joint United Nations Programme on HIV/AIDS (UNAIDS), WHO, Geneva, Switzerland, 2010.

[3] Central Statistical Agency and ICF Macro, Ethiopia Demographic and Health Survey, ICF Macro, Calverton, Md, USA, 2011.

[4] W. Deressa, A. Seme, A. Asefa, G. Teshome, and F. Enqusellassie, "Utilization of PMTCT services and associated factors among pregnant women attending antenatal clinics in Addis Ababa, Ethiopia," BMC Pregnancy and Childbirth, vol. 14, article 328, 2014.

[5] G. N. Nkuoh, D. J. Meyer, P. M. Tih, and J. Nkfusai, "Barriers to men's participation in antenatal and prevention of motherto-child HIV transmission care in Cameroon, Africa," Journal of Midwifery and Women's Health, vol. 55, no. 4, pp. 363-369, 2010.

[6] WHO, Preventing Mother-to-Child Transmission of HIV to Reach the UNGASS and MDGs Moving towards the Elimination of Pediatric HIV. PMTCT Strategic Vision 2010-2015, WHO, Geneva, Switzerland, 2011.

[7] A. Adera, M. Wudu, Y. Yimam, S. Mengistie, M. Kidane, and A. Woreta, "Factors that affects male partner involvement in PMTCT services in africa: a review literature," Science Journal of Public Health, vol. 3, no. 4, pp. 460-467, 2015.

[8] F. Haile and Y. Brhan, "Male partner involvements in PMTCT: a cross sectional study, Mekelle, Northern Ethiopia," BMC Pregnancy and Childbirth, vol. 14, article 65, 2014.

[9] J. Homsy, J. Obonyo, J. Ojwang et al., "Routine intrapartum HIV counseling and testing for prevention of mother-to-child transmission of HIV in a rural Ugandan hospital," Journal of Acquired Immune Deficiency Syndromes, vol. 42, no. 2, pp. 149154, 2006.

[10] I. Thior, S. Lockman, L. M. Smeaton et al., "Breastfeeding plus infant zidovudine prophylaxis for 6 months vs formula feeding plus infant zidovudine for 1 month to reduce mother-to-child HIV transmission in Botswana: a randomized trial: the Mashi Study," The Journal of the American Medical Association, vol. 296, no. 7, pp. 794-805, 2006.

[11] J. Orne-Gliemann, P. T. Tchendjou, M. Miric et al., "Coupleoriented prenatal HIV counseling for HIV primary prevention: An Acceptability Study," BMC Public Health, vol. 10, article 197, 2010.

[12] M. Tilahun and S. Mohamed, "Male partners' involvement in the prevention of mother-to-child transmission of HIV and associated factors in Arba Minch Town and Arba Minch Zuria Woreda, Southern Ethiopia," BioMed Research International, vol. 2015, Article ID 763876, 6 pages, 2015.

[13] A. Endawoke, T. Gebeyaw, and A. Amanuel, "Level of male partner involvement and associated factors in prevention of mother to child transmission of HIV/AIDS services in Debremarkos town, Northwest Ethiopia," African Journal of AIDS and HIV Research, vol. 1, no. 2, pp. 16-25, 2013.

[14] D. Getu, Factors Related to Male Participation in Prevention of Mother-to-Child Transmission of Human Immunodeficiency Virus in Three Public Hospitals in Addis Ababa, Ethiopia, University of South Africa, 2011.
[15] B. K. F. Mohlala, M.-C. Boily, and S. Gregson, "The forgotten half of the equation: randomized controlled trial of a male invitation to attend couple voluntary counselling and testing," AIDS, vol. 25, no. 12, pp. 1535-1541, 2011.

[16] I. T. Kamal, "Field experiences in involving men in safe motherhood," Report of the Meeting of WHO Regional Advisers in Reproductive Health, WHO, Washington, DC, USA, 2001.

[17] O. Shisana, T. Rehele, L. C. Simbayi et al., South African National HIV Prevalence, HIV Incidence, Behavior and Communication Survey, Cape Town HSRC Press, 2005.

[18] Z. Pemberai, C. Hope, S. Chiedzwa, and D. Rumbidzai, Understanding factors that cause low male involvement in Community HIV programs for effective design of gender inclusive programs, An operations research report submitted to the regional AIDS training network family and AIDS caring trust (fact) research and knowledge management Department, Zimbabwe, 2011.

[19] F. M. Bwambale, S. N. Ssali, S. Byaruhanga, J. N. Kalyango, and C. A. S. Karamagi, "Voluntary HIV counselling and testing among men in rural western Uganda: implications for HIV prevention," BMC Public Health, vol. 8, article 263, 2008.

[20] Department of Reproductive Health and Research, Male Involvement in the Elimination of Mother-to-Child Transmission of HIV, Department of Reproductive Health and Research, Geneva, Switzerland, 2011.

[21] D. A. Katz, J. N. Kiarie, G. C. John-Stewart, B. A. Richardson, F. N. John, and C. Farquhar, "Male perspectives on incorporating men into antenatal HIV counseling and testing," PLoS ONE, vol. 4, no. 11, Article ID e7602, 2009.

[22] T. Dinzela, Factors influencing men's involvement in prevention of mother to child transmission (PMTCT) of HIV programmes in Mambwe District, Zambia [M.S. thesis], University of South Africa, 2006. 


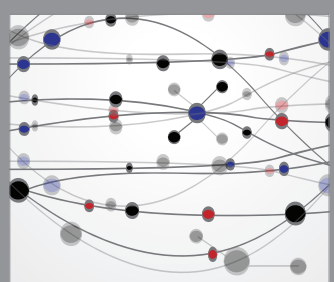

The Scientific World Journal
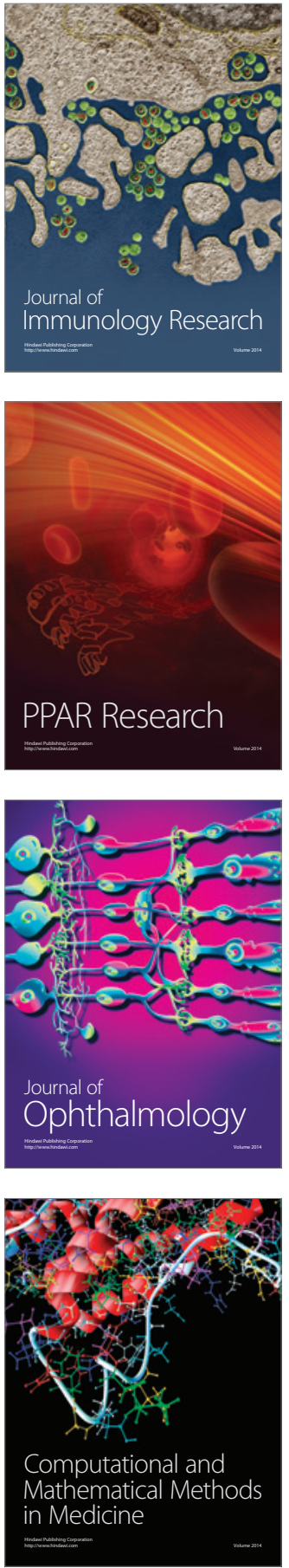



Gastroenterology Research and Practice

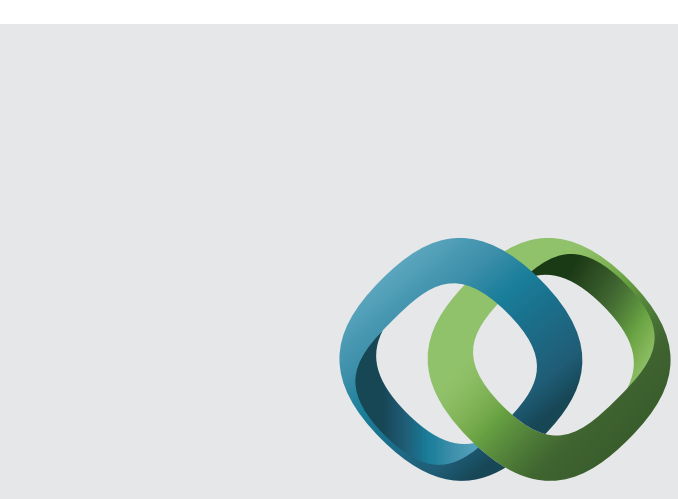

\section{Hindawi}

Submit your manuscripts at

http://www.hindawi.com
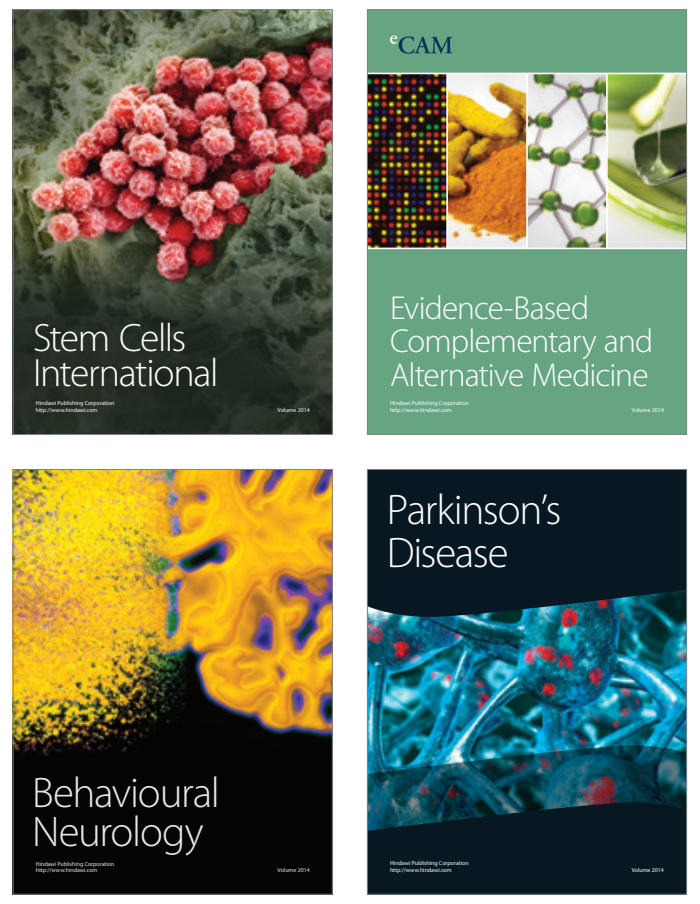
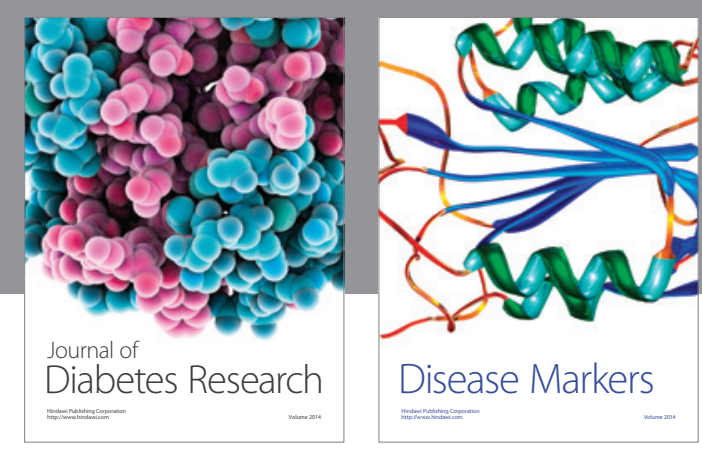

Disease Markers
